\title{
Etiological Diagnosis of Non-Traumatic Myelopathies: Experience in a Tertiary Health Care Center in Bangladesh
}

\author{
NC KUNDU $^{\mathrm{a}}$, FA QURAISHI ${ }^{\mathrm{b}}, \mathrm{M} \mathrm{SEN}^{\mathrm{c}}$
}

Summary:

Background: The relative frequency of non-traumatic compressive and non-compressive myelopathies and their etiologies have not been evaluated extensively in Bangladesh. This study was aimed to identify the etiological diagnosis of non-traumatic myelopathies.

Methods: One hundred cases of non-traumatic myelopathic admitted patients were prospectively studied during 20092012 in the neurology ward of Sir Salimullah Medical College and Mitford Hospital, Dhaka, Bangladesh. Patients underwent a detailed clinical evaluation followed by laboratory investigations and neuroimaging studies.

Results: Among 100 cases, 46 patients (male 41 and female 5) presented with quadriparesis and 54 patients (male 30 and female 24) presented with paraparesis. Duration of symptoms before presentation to the hospital was

\section{Introduction:}

The term myelopathy describes pathologic conditions that cause spinal cord, meningeal or perimeningeal space damage or dysfunction. Based on the Sicard and Forstier classification, myelopathies are divided into compressive and non-compressive, in relation to subarachnoid space obstruction. There are cases where the etiology is never identified, and they are classified as idiopathic myelopathy. Compressive diseases of the spinal cord divided into acute and chronic, including degenerative changes, trauma, tumor infiltration, vascular malformations, infections with abscess formation, and syringomyelia. Non-compressive

a. Dr. Narayan Chandra Kundu. Associate Professor, Neurology, Shaheed Suhrawardy Medical College. Sher-E- Bangla Nagar, Dhaka- 1207.

b. Prof. Firoz Ahmed Quraishi, Professor, Neurology, Anwer Khan Modern Medical College, Dhanmondi- 7, Dhaka.

c. Dr. Moushumi Sen, Associate Professor, Biochemistry, Anwer Khan Modern Medical College, Dhanmondi- 7, Dhaka.

Address of Correspondence: Dr. Narayan Chandra Kundu, Associate Professor, Neurology, Shaheed Suhrawardy Medical College, Sher-EBangla Nagar, Dhaka-1207, Mobile: 01711105369.

Received: 19 March 2017

Accepted: 20 Jan. 2018 considerably higher in quadriparesis compared to paraparesis $(230.09 \pm 31.16$ days VS 136.82 \pm 21.77 days; p value $=0.016$ ). There were no significant differences in the number of patients admitted between compressive and non-compressive groups (p value - 0.4035). Common etiologies observed in this study were vertebral disc disease (23\%), spinal cord tumor (15\%), Pott's disease (15\%), acute transverse myelitis (13\%) and motor neuron disease (11\%).

Conclusion: Vertebral disc disease, spinal cord tumors, infection (Pott's disease), post infectious condition (ATM), and neurodegenerative disease (MND) are mostly responsible for myelopathies.

Keywords: Non traumatic, Etiology, Paraplegia/ Quadriplegia.

(J Bangladesh Coll Phys Surg 2018; 36: 53-58) DOI: http://dx.doi.org/10.3329/jbcps.v36i2.36066

myelopathy encompasses a large range of disease entities ranging from demyelination, infection, nutritional, toxic, and heredo-familial to degenerative conditions. The disease spectrum varies and is somewhat different in Asian countries as compared to western and African countries ${ }^{1}$. Infections and nutritional diseases are common in this part of the world while demyelinating and HIV associated diseases are common in western and African countries respectively. Quadriparesis and paraparesis due to nontraumatic myelopathies are common neurological diseases with high morbidity (up to $79 \%$ of patients will definitely remain disabled) and mortality ${ }^{1-3}$. Diseases affecting the spinal cord and complicated by neurological damage are important health problem in Bangladesh as they carry high rates of morbidity and mortality. With the advent of MRI, which is a very sensitive modality of investigation for the intramedullary spinal lesions and availability other investigations, it has become pertinent to have a relook at the profiles of non-traumatic myelopathies as many of the processes affecting the spinal cord may be reversible, if they are recognized and treated early. An increasing understanding of the underlying etiological 
factors will be beneficial in managing spinal cord diseases more comprehensively.

\section{Material and Methods:}

This cross sectional prospective observational study conducted on one hundred (100) patients with nontraumatic myelopathies admitted in the neurology ward of Sir Salimullah Medical College and Mitford Hospital during the period of 2009-2012. They were examined clinically and followed up by laboratory investigations and neuroimaging studies. Details of onset of illness, antecedent events, progression and symmetry/asymmetry of symptoms and findings of clinical examination were recorded in a structured format. Patients who fulfilled the inclusion and exclusion criteria were enrolled. A diagnosis of myelopathy was arrived at using the diagnosis algorithm.

Patients were classified as having compressive (CM) or non-compressive (NCM) myelopathies based on MRI findings. Routine investigations including complete blood count (CBC) with erythrocyte sedimentation rate (ESR), X-ray chest (CxR) (P/A), urine for routine examination, random blood sugar (RBS), and ultra-sonogram of whole abdomen were done in all patients. MRI of region of interest and extended screening was done whenever it was thought to be logical. Cerebrospinal fluid (CSF) study (routine) was done in selected cases. IgG index and oligoclonal band and serum aquaporin-4 antibodies (AQP4-Ab) were not done due to lack of facilities and economic constrain. Nerve conduction study (NCS and EMG) was done in appropriate case. Estimation of vitamin B12 level, rheumatoid factor, ANF, anti-ds DNA, VDRL (including CSF VDRL) and anti HIV antibody was done wherever suspected. Surface and CT guided fine needle aspiration cytology (FNAC) was done in selected cases. Upper gastrointestinal endoscopy and biopsy was done in cases of vitamin B 12 deficiency. Mantoux test (MT) and Slit Skin Smears (SSS) test were done as additional investigations for presence of Mycobacterium in suspected cases. Bone marrow study and other relevant investigations were done in few cases in consultation with the Hematologist.

Collected data were analyzed by using T-test, Fisher's exact test and considered significant with $\mathrm{p}$ value $<0.05$.

\section{Results:}

Age and sex distribution of 100 patients were presented in Table-1. Among 100 patients, 46 patients (male 41 and female 5) had quadriparesis and 54 patients (male 30 and female 24) presented with paraparesis and breakup of etiological diagnosis was showed in Table-2.

Duration of symptoms before presenting to this hospital varied widely and ranged from 1 day to 2 years or more. The mean disease duration before presentation was considerably higher in patients diagnosed with quadriparesis (230.09 days \pm 31.16 days) compared to that of paraparesis (136.82 days \pm 21.77 days) and the difference was statistically significant $(\mathrm{p}<0.05)($ Table-3). A further analysis showed the duration was significantly higher for quadriparesis compared to that of paraparesis $(p<0.05)$ (Table-4) in non-compressive cases. The mean duration was also higher for quadriparesis in compressive cases; however, the difference was statistically insignificant.

Among the patients with quadriparesis, presence of compressive lesion was higher compared to noncompressive disorder [(58.7\% VS 41.3\%) (Table-5)] . Similarly, compressive lesions accounted for the majority of paraparesis cases compared to noncompressive disorder (70.4\% VS 29.6\%). Overall, there were more cases of compressive disorder (Table2 and Table- 5) which seemed to pose a slightly higher risk $(\mathrm{RR}=1.22)$ of being diagnosed with paraparesis, although, the difference was not statistically significant $(\mathrm{p}=0.4035)$.

Among the compressive quadriparesis patients, 19 $(70.37 \%)$ patients were due to cervical disc disease and $3(11.11 \%)$ due to tuberculous disease of the spine. Spinal cord tumors and syringomyelia were responsible in remaining cases. For quadriparesis, the most important non-compressive cause was found to be motor neuron disease (MND) (57.89\%) followed by acute transverse myelitis $(21.05 \%)$. Sub acute combined spinal cord disease (SACD), Devic's disease and hereditary causes were the contributors in the remaining cases $(21.10 \%)$.

Pott's disease, also known as tuberculous Spondylitis, was the most common [12 (32.43\%)] compressive disorder to cause paraparesis. Spinal cord tumors were equally responsible for paraparesis (11/37). NonHodgkin's lymphoma came out to be an important cause 
for compressive spastic paraparesis [4 (10.81\%)]. Similar numbers of cases was due to metastatic spine disease. Disc disease was rather uncommon [4 $(10.81 \%)]$. On the other hand, acute transverse myelitis [9 (52.94\%)] was the most common non-compressive cause accounted for paraparesis. In 4 (23.53\%) patients no definite diagnosis could be reached by conventional investigations.

Interestingly, spinal cord tumors including metastatic spinal cord diseases were significantly higher in paraparesis compared to quadriparesis (15 VS 4) and the difference was statistically significant (Fisher's exact test, $\mathrm{p}<0.05$ ). On the contrary, vertebral disc disease was more predominant in quadriparesis compared to paraparesis where the difference was very highly significant $(\mathrm{p}<0.001)$. In addition, both Pott's disease and acute transverse myelitis were more common in paraparesis although the difference was significant only for Pott's disease $(\mathrm{p}<0.5)$ (Table-6).

In three cases symptoms and clinical investigation results were suggestive of sub acute combined degeneration of spinal cord (one presented with quadriparesis and two with paraparesis). All three were male and none of them were pure vegetarian.

Rest of the conditions producing non-compressive myelopathies (paraparesis and quadriparesis) formed a small percentage and included 6 cases of hereditary and unclassified upper motor type lesion.

Syringomyelia, epidural abscess and parasagittal lesions were responsible for 3 cases (one for each) of compressive myelopathy.

Table-I

\begin{tabular}{llcccccc}
\multicolumn{7}{c}{ Age and Sex distribution of study population } \\
Sex & Weakness & $<20$ yrs. & $21-30$ yrs. & $31-40$ yrs. & $41-50$ yrs. & $51-60$ yrs. & $>60$ yrs. \\
\hline M & Paraparesis & 6 & 8 & 5 & 6 & 4 & 1 \\
& Quadriparesis & 1 & 6 & 8 & 11 & 11 & 4 \\
F & Paraparesis & 1 & 3 & 8 & 5 & 6 & 1 \\
& Quadriparesis & 1 & 2 & 0 & 2 & 0 & 0 \\
\hline
\end{tabular}

Table-II

\begin{tabular}{llccc}
\multicolumn{5}{c}{ Etiological diagnosis of paraparesis and quadriparesis $(N-100)$} \\
Etiology main group & Etiology subgroup & Paraparesis $(\mathrm{N})$ & Quadriparesis $(\mathrm{N})$ & Total (N) \\
\hline Compressive & Vertebral disc disease & $4(10.81 \%)$ & $19(70.37 \%)$ & 23 \\
& Spinal cord tumors & $11(29.73 \%)$ & $4(14.81 \%)$ & 15 \\
& Pott's disease & $12(32.43 \%)$ & $3(11.11 \%)$ & 15 \\
& Metastases & $4(10.81 \%)$ & 0 & 4 \\
& Non-Hodgkin's Lymphoma & $4(10.81 \%)$ & 0 & 4 \\
\multirow{5}{*}{ Subtotal } & Others & $2(5.40 \%)$ & $1(3.70 \%)$ & 3 \\
Non-compressive & Motor neuron disease (MND) & $37(100 \%)$ & $27(100 \%)$ & 64 \\
& Acute transverse myelitis & 0 & $11(57.89 \%)$ & 11 \\
& No definite cause & $4(23.53 \%)$ & $4(21.05 \%)$ & 13 \\
Subtotal & Others & $4(23.53 \%)$ & $2(10.52 \%)$ & 6 \\
\hline Total & & $17(100 \%)$ & $19(100 \%)$ & 6 \\
\hline
\end{tabular}

Note:

3 patients of sub acute combined degeneration of spinal cord, one each of syringomyelia, epidural abscess, parasagittal meningioma and anterior two third syndrome (vascular) included in other causes (both compressive and non-compressive). 
Table-III

Duration of symptoms before presentation between paraparesis and quadriparesis

\begin{tabular}{lcc} 
Data analyzed & Mean \pm SEM (days) & p value \\
\hline Paraparesis & $136.82 \pm 21.77$ & 0.0162 \\
Quadriparesis & $230.09 \pm 31.16$ & \\
\hline
\end{tabular}

Note: SEM- Standard error of Mean

t- Test: Two- Sample assuming unequal variance. Difference between paraparesis and quadriparesis is significant $(\mathrm{p}<0.05)$

Table-IV

Duration of symptoms before presentation between compressive and non-compressive group

\begin{tabular}{lccc}
\multirow{2}{*}{ Etiology } & \multicolumn{2}{c}{ Type of myelopathy } & p value \\
\cline { 2 - 3 } & Paraparesis & Quadriparesis & \\
\hline Compressive & $149.00 \pm 28.6$ days & $215.40 \pm 42.9$ days & 0.204 \\
Non-compressive & $110.2 \pm 30.10$ days & $250.90 \pm 45.4$ days & 0.015 \\
\hline
\end{tabular}

t- Test: Two- Sample assuming unequal variance. Difference between paraparesis and quadriparesis is significant $(\mathrm{p}<0.05)$

Table-V

Compressive VS non-compressive causes of myelopathies

\begin{tabular}{lcccc} 
Data analyzed & Paraparesis & Quadriparesis & Total & p value \\
\hline Compressive & $37(70.4 \%)$ & $27(58.7 \%)$ & 64 & 0.4035 \\
Non-compressive & $17(29.6 \%)$ & $19(41.3 \%)$ & 36 & \\
\hline
\end{tabular}

Fisher's exact test comparing compressive VS non-compressive causes of myelopathies. Significance at $\mathrm{p}$ value $<.05$.

Table-VI

Subgroup analysis of etiological diagnosis in two types of myelopathies (Paraparesis VS Quadriparesis)

\begin{tabular}{lccccc} 
Data analyzed & Paraparesis & Quadriparesis & Total & p value & $95 \%$ CI \\
\hline Spinal cord tumors & 16 & 04 & 20 & 0.0117 & $1.225-2.315$ \\
Pott's disease & 12 & 03 & 15 & 0.468 & $1.161-2.257$ \\
Vertebral disc disease & 04 & 19 & 23 & $<0.0001$ & $0.1082-0.662$ \\
Acute transverse myelitis & 09 & 04 & 13 & .3717 & $.8834-2.028$ \\
\hline
\end{tabular}

Fisher's exact test comparing subgroup analysis of etiological diagnosis between compressive VS non-compressive causes of myelopathies. Level of significance ( $\mathrm{p}$ value $<.05$ ).

\section{Discussion:}

Quadriparesis and paraparesis are conditions with considerable morbidity having tremendous social repercussions. They bring about endless and constant misery to the patients, family and the society.
In this series paraparesis was almost equally prevalent between 20-50 years of age while quadriparesis was more common between 41- 60 years in male population. This may be due to higher prevalence of motor neuron disease in those age groups. On the other 
hand, most of the female presented with paraparesis between 31-60 years of age and no clusters were noted for quadriparesis.

Duration of symptoms before presenting to this hospital was different from few days to 2 years or more. The mean disease duration before presentation was considerably higher in patients diagnosed with quadriparesis $(230.09 \pm 31.16)$ compared to that of paraparesis $(136.82 \pm 21.77)$ and the difference was statistically significant $(p<0.05)$. This is much higher than studies done in some African countries ${ }^{4,15-16}$. The possible reasons are different patients setting and poor disease understanding both on the part of primary care givers and patients.

It is estimated that involvement of the spine occurs in less than $1 \%$ of patients with tuberculosis. Tuberculosis was one of the commonest causes of compressive paraparesis in this series and was diagnosed in 12 cases $(32.43 \%)$ while in quadriparesis it was seen in only in 3 cases $(11.11 \%)$. In three separate studies reported in Africa in 1995, 1995 and 2011 and one study from India in 2006, tuberculosis was the leading cause of paraparesis accounting for $29.69 \%, 47 \%, 44.9 \%$ and $33.33 \%$ cases respectively ${ }^{5-8}$. However, a study by Zingraff LLA et al in 2010 in Yaounde (Cameroon) reported that only $12.9 \%$ cases of myelopathy were due to spinal tuberculosis ${ }^{9}$, which is quite a lower incidence that the present series and the mentioned studies. The involvement of lower thoracic spine (T7T12) was seen in $75 \%$ cases while upper thoracic spine in $25 \%$ cases. This is just opposite to a study by Chaurasia $\mathrm{RN}$ et al where involvement was more in upper thoracic spine ${ }^{4}$.

Disc disease was responsible in $70.37 \%$ cases to cause quadriparesis and when compared with paraparesis the difference was statistically highly significant ( $\mathrm{p}<0.001)$. It is higher than that observed in a study by Chaurasia $\mathrm{RN}$ et al ${ }^{4}$, and this higher incidence may be due to manual nature of work of the study population.

ATM is a monophasic illness and represents a localized form of post infectious encephalomyelitis. In this study acute transverse myelitis is more likely to cause paraparesis than quadriparesis [9 (56.94\%) cases VS $4(21.05 \%)$ cases] but not statistically significant. Overall incidence of ATM causing quadriparesis and paraparesis was $13 \%$ and compatible with the findings of Chaurasia $\mathrm{RN}$ et al ${ }^{4}$.
Spinal cord tumors including metastases occur more commonly in dorsal spine than in cervical region. Overall number of patients was 19 [19\%, 15 and 4 cases for paraparesis and quadriparesis respectively)]. This is in agreement with many studies where its incidence varies from 21-30\% of all compression 4, 11-13. However this was substantially lower when compared with a study done by Zingraff LLA et al ${ }^{9}$, where its incidence was about $49 \%$ and was the leading cause of compressive myelopathy. In the present study, primary spinal cord tumors were diagnosed in 15 patients while secondary causes comprised of 4 cases.

4 cases of Non-Hodgkin's lymphoma were diagnosed and of which 2 patients were previously treated for Pott's disease.

Full blown clinical picture of vitamin B12 deficiency consists of macrocytic anemia, atrophic glossitis, peripheral and central neurological disorders ${ }^{14}$. In this series 3 cases were diagnosed as SACD of spinal cord. We were not able to do serum methyl malonic acid and serum intrinsic factor antibody. Early suspicion and relevant investigations and early treatment are essential to prevent irreversible damage.

In 6 cases $(6 \%)$ no definite etiological diagnosis could be reached and leveled as heredo- familial (where possible) and unclassified upper motor lesion. This is due to lack of advance technological support including chromosomal studies.

\section{Conclusion:}

Compressive causes are commonly responsible for myelopathy and many of these could be make reversible by early surgical intervention. This study revealed that duration of symptoms is long and this may lead to a noncurable state even after surgery. On the other hand motor neuron disease was also presented late. Awareness of patients and attending doctors are essential for early recognition and treatment. Extension of investigation facilities in divisional level and trained man power are also equally important to handle these groups of patient.

\section{References:}

1. Prabhakar S, Syal P, Lal V, Khadelwal N, Das CP. Non-compressive myelopathy: clinical and radiological study. Neurol India 1999; 47: 294

2. Kim RY, Spencer SA, Meredith RF. Extradural spinal cord compression: analysis of factors determining functional prognosis-prospective study. Radiology. 1990; 1762: 279-82. 
3. Scrimgeour EM. Non-traumatic paraplegia in northern Tanzania. BMJ 1981;283: 975-80.

4. Chaurasia RN, Verma A, Joshi D, Misra S. Etiological spectrum of non-traumatic myelopathies: experience from a tertiary care centre. JAPI. 54; 2006: 445-48.

5. Zenebe G. Myelopathies in Ethiopia. East Afr Med J 1995; 72 : $42-45$.

6. Nyame PK. An etiological survey of paraplegia in Accra. East Afr Med J 1994; 71: 527-30.

7. Zenbe G, Oli K, Tekel-Haimanto R. Paraplegia at the Tikur Anbessa Teaching Hospital: A seven year retrospective study of 164 cases. Ethiop Med J 1995; 33: 7-13.

8. Owolabi LF, Ibrahim A, Samaila AA. Profile and outcome of nontraumatic paraplegia in Kano, northwestern Nigeria. Ann Afr Med 2011; 10: 86-90

9. Zingraff LLA, kengne AP, Djienteceu Vincent de Paul, Kaute CT, Nijamnshi AK. Patterns of non-traumatic myelopathies in
Yaounde (Cameroon): a hospital based study. J Neurol Neurosurg Psychiatry 2010; 81: 768-770

10. Saleh M, Deeb Al, Basim A. Acute transverse myelitis- A localized form of post-infectious encephalomyelitis. Brain 1997; 7: 1115-22.

11. Mehrotra AN, Khosla SN, Pathak LR. Spinal paraplegias. JAssoc Physicians India 1966; 14: 121-28.

12. Chaudhary KL, Kulpati Bhargava SP, Mittal MM Sharma ML. Etiology of paraplegia. J Assoc Physicians India 1968; 16: 751-58.

13. Mani KS, Punekar BD, Rao J, Nair SD. Etiology of paraplegia. Neurology India 1966; 15: 19-26.

14. Toh BH, Van Driel IR, Glesson PA. Pernicious anemia. N Engl J Med 1997; 337: 144-48.

15. Abraham JL. Assessment and treatment of patients with malignant spinal cord compression. J Support Oncol 2004; 2: $377-401$.

16. Chamberlain MC, Kormanik PA. Epidural spinal cord compression. Neuro Oncol 1999. 120-23. 